\title{
Uso tradicional de plantas medicinales con acción diurética en el Municipio de Quemado de Güines, Cuba
}

\author{
Maykel Pérez Machín ${ }^{1}$, Mario L. Sueiro ${ }^{2}$, Ania de la Cruz ${ }^{3}$, María A. Boffill ${ }^{1}$, Francisco Morón ${ }^{4}$, \\ Orestes R. Méndez ${ }^{5}$ \& Jaqueline Cárdenas ${ }^{1}$ \\ 1. Vicerrectorado Académico, Universidad de Ciencias Médicas de Villa Clara, Carretera a Acueducto y Circunvalación, \\ C.P.50200, Santa Clara, Cuba; maykelpm@ucm.vcl.sld.cu, mariaboffill@yahoo.com,jaquelinecs@ucm.vcl.sld.cu \\ 2. Facultad de Química-Farmacia, Departamento de Farmacia. Universidad Central "Marta Abreu" de Las Villas, \\ Carretera de Camajuaní, Km 51⁄2, Santa Clara, Villa Clara, Cuba; msueiro@uclv.edu.cu \\ 3. Sede Universitaria Municipal de Qüemado de Güines, Calzada 26 de Julio s/n, C.P.52200. \\ 4. Laboratorio Central de Farmacología, Universidad de Ciencias Médicas de La Habana, Facultad "Dr. Salvador \\ Allende", La Habana, Cuba; moron@infomed.sld.cu \\ 5. Centro de Estudios Jardín Botánico de Villa Clara, Facultad de Ciencias Agropecuarias, Universidad Central "Marta \\ Abreu" de Las Villas, Carretera de Camajuaní, Km 5ำ2, Santa Clara, Villa Clara, Cuba; omendezo@uclv.edu.cu
}

$$
\text { Recibido 21-IX-2010. C Corregido 03-III-2011. Aceptado 30-III-2011. }
$$

\begin{abstract}
Tradicional use of medicinal plants with diuretic properties at Quemado de Güines Municipality, Cuba. Medicinal plants are highly rich in Cuba and an amount of 179 species have been reported to be used by the population for diuretic purposes, nevertheless, no experimental validation has supported this effect. This study presents the relative importance of the medicinal plant species most widely used for diuretic purposes in two communities of Quemado de Guines Municipality, Villa Clara province. The information was obtained through the application of an interview to 85 inhabitants, from which 80 were random surveys to people with a great knowledge of plants, and five to herbalists and doctors practicing natural medicine. The etnopharmacological information was registered (gathered) by means of the "Tradicional of the Medicine of the Island" (TRAMIL) methodology and the interesting species were identified by a botanist and deposited in the Herbarium of the Central University "Marta Abreu" from Villa Clara, registered in the Index Herbarium, published periodically by the International Association for Plant Taxonomy. The data was analyzed by means of the indexes of use values and significant use level after TRAMIL. From the total of 19 botanical families, 26 medicinal species were identified, and 10 plants resulted with higher significant use and higher indexes of use values. From the plants reported as diuretics, 53.8\% have not been experimentally validated in Cuba, the rest of the identified species have been validated at a preclinical level in some centers in the country, but its use have not been authorized as phytochemicals by the Cuban Regulatory Agency. The documentation related to the use of medicinal plants in the studied areas reveals that the traditional knowledge continues deeply rooted in the communities, and popular wisdom is kept through the representative images of the herbalist and people with considerable knowledge about this topic. Rev. Biol. Trop. 59 (4): 1859-1867. Epub 2011 December 01.
\end{abstract}

Key words: ethnobotanic, medicinal plant, diuretic, traditional knowledge, Quemado de Güines.

Los diuréticos son fármacos que estimulan la excreción renal de agua y electrólitos, como consecuencia de su acción perturbadora sobre el transporte iónico a lo largo de la nefrona. Esta interferencia puede llevarse a cabo en uno o varios sitios del recorrido tubular y su objetivo fundamental es conseguir un balance negativo de agua, pero los diuréticos no actúan directamente sobre el agua, sino a través del sodio (diuréticos natriuréticos) o de la osmolaridad (diuréticos osmóticos). De acuerdo con ello, la finalidad principal de los diuréticos se dirige al tratamiento de los edemas. Sin embargo, directa o indirectamente pueden modificar 
otros iones y alterar otras funciones, de ahí que se utilicen también en otras enfermedades, como la hipertensión arterial, las hipercalcemias, la diabetes insípida, el glaucoma, las intoxicaciones, entre otras (Flórez 2003, Rang et al. 2008). Existe un amplio arsenal terapéutico de fármacos sintéticos que son empleados con estos fines; no obstante, nuestra población utiliza para ello un número considerable de decocciones e infusiones de plantas medicinales, que se han transmitido de generación en generación mediante un enfoque etnobotánico (Pimentel 2009).

Se calcula de manera general que el $64 \%$ de la población mundial hace uso en forma no industrializada de las plantas medicinales, ya sea de sus partes enteras o en forma de infusiones. En países tecnológicamente avanzados como los Estados Unidos un $60 \%$ de su población emplea habitualmente plantas medicinales para combatir ciertas dolencias y en Japón hay más demanda de preparados de plantas medicinales que de medicamentos oficiales (Macías 2009).

Esta práctica de uso está asociada al empirismo en muchos casos, y faltan estudios químicos, preclínicos, clínicos y epidemiológicos que confirmen de forma fehaciente los efectos farmacológicos de las plantas y los principios activos responsables del efecto (González \& Ramírez 2007). Existen diversos estudios que deben realizarse antes de utilizar en seres humanos un nuevo fitomedicamento; sin dudas no se puede exigir lo mismo a una nueva molécula sintetizada y que nunca el hombre ha estado expuesto a ella, con total desconocimiento de sus acciones y efectos adversos, que a una planta medicinal administrada de forma tradicional durante muchos siglos y tal vez empleada en múltiples países. Esto no significa que podamos confiarle a la sabiduría popular la seguridad y eficacia de las plantas, pues ellas poseen numerosas sustancias con actividad biológica, capaces potencialmente de producir cualquier efecto indeseable, muchos de los cuales pueden pasar inadvertidos hasta en una investigación adecuadamente diseñada (Morón 2002).
En el año 1998 los editores del New England Journal Medicine declararon: "Es tiempo de que la comunidad científica detenga el libre andar de la medicina alternativa" (Marcia et al. 2005). Entre otros elementos, su planteamiento estaba fundamentado en las pocas publicaciones científicas en este campo. A partir de esta declaración los practicantes e investigadores de este tipo de medicina comenzaron a defenderla realizando publicaciones al respecto, las que aún son insuficientes, pues muchos de los fitomedicamentos que se comercializan adolecen de todos los estudios preclínicos establecidos (Pérez et al. 2007).

En Cuba, la introducción de la Medicina Tradicional comienza en el siglo XV, pero no es hasta mediados del siglo XX que alcanza su máxima expresión con el insigne Doctor Juan Tomás Roig, quién identifica 595 especies de plantas medicinales empleadas por la población cubana para diferentes usos curativos y hace un llamado a la comunidad científica nacional para que se estudien las mismas con el objetivo de comprobar su seguridad y eficacia, planteando además la necesidad de desarrollar la industria nacional (Roig 1998, González \& Ramírez 2007).

En años recientes se ha propuesto la combinación de métodos y técnicas propias de la investigación cuantitativa y cualitativa que permitan seleccionar las plantas más usadas por la población para validar su uso tradicional.

Los estudios etnobotánicos han tomado una gran relevancia en los últimos años, ya que varias compañías farmacéuticas están interesadas en las plantas como un gran potencial para la obtención de fármacos de origen natural útiles en el tratamiento de las enfermedades que más afectan a las comunidades (Bermúdez \& Velásquez 2002). Cada día se presta más atención al estudio de las especies medicinales de manera tal que la etnobotánica, la fitoterapia y la fitoquímica están tomando un auge, tanto en la práctica de la medicina complementaria como en el ámbito académico (Verpoorte et al. 2005, Gauniyal 2005).

Algunos investigadores han tratado de desarrollar metodologías que permiten 
cuantificar la información etnobotánica a través de la estimación del valor de uso de las plantas, utilizando la información obtenida en las comunidades encuestadas basándose en exámenes sistemáticos que incluyen a un determinado número de informantes. Los datos de cada informante son usados para calcular el número de aplicaciones de cada una de las especie en particular (Pérez et al. 2011).

El objetivo del presente estudio fue evaluar a través de la etnobotánica, el estado actual del conocimiento tradicional sobre plantas medicinales con actividad diurética atribuida en comunidades del municipio de Quemado de Güines.

\section{MATERIALES Y MÉTODOS}

El área del presente estudio incluyó las comunidades nombradas como barrios La Puya y El Perejil del municipio villaclareño de Quemado de Güines. El mismo se ubica entre los 20 asentamientos más antiguos de Cuba, situado al norte de la provincia de Villa Clara a $72 \mathrm{~km}$ de la ciudad de Santa Clara, con una extensión territorial de $340.2 \mathrm{~km}^{2}$ y una población de 22515 habitantes. La existencia en este territorio de áreas rurales extensas, con una población arraigada a las costumbres de antaño, conservadora de usos tradicionales, hace posible que puedan llevarse a cabo estudios etnobotánicos con éxito.

El estudio etnobotánico en dichas comunidades consideró una muestra intencional no probabilística conformada por un total de 85 habitantes. Se aplicó aleatoriamente 80 encuestas a conocedores de plantas, y se realizó cinco entrevistas a yerberos y médicos practicantes de medicina tradicional de la localidad. Los grupos muestrales, considerados heterogéneos entre sí, quedaron conformados a partir de su conocimiento homogéneo sobre el uso de las plantas medicinales, considerando el criterio popular o el saber científico.

En una primera acción de la investigación etnobotánica, se realizó una revisión documental sobre los principales trabajos científicos que abordan el tema de las plantas diuréticas utilizando para ello las siguientes categorías de búsqueda (plantas diuréticas cubanas, reporte etnomédico y validación farmacológica). En una segunda actividad, se aplicó la entrevista a los yerberos (informantes claves), para identificar las plantas medicinales utilizadas de forma tradicional con estos fines. Como tercera actividad, se aplicó un cuestionario a conocedores de plantas siguiendo la metodología establecida por Tradicional of the Medicine of the Island (TRAMIL) (TRAMIL 2006) y según las sugerencias de otros autores (Martin 1995). Finalmente, se realizó una entrevista focalizada a médicos practicantes de medicina tradicional para diferenciar el saber científico del popular. Se utilizó la triangulación como metodología fundamental de análisis con el propósito de contrastar y establecer un control cruzado de los datos obtenidos. Este proceso nos permitió hacer un descarte de las no coincidencias (conocimiento refinado) para obtener las principales plantas utilizadas como diuréticas por la población que carecen de validación experimental en Cuba y que necesitan ser estudiadas desde el punto de vista científico.

Las plantas recolectadas durante las encuestas y entrevistas se trataron según técnicas de herborización para muestras botánicas, las cuales se depositaron en el Herbario "Dr. Alberto Alonso Triana" (ULV) del Jardín Botánico de la Universidad Central "Marta Abreu" de Las Villas, el cual aparece registrado en el Index Herbariorum, que se publica periódicamente por la Internacional Association for Plant Taxonomy. Se separaron los ejemplares pertenecientes a la serie ULV y a la serie Instituto Superior Pedagógico de Villa Clara (HPVC) encontrándose ésta última formando parte de la colección de la referida Universidad. Las especies ya depositadas en el herbario fueron comparadas con los especímenes históricos y los registros no disponibles fueron depositados por el especialista en la serie ULV con su número correspondiente. Las respuestas proporcionadas por los informantes se organizaron en una base de datos utilizando Microsoft Excel ${ }^{\circledR}$. Se calculó el Índice de Valor de Uso (IVU) y el Nivel de Uso Significativo TRAMIL 
(UST) para cada una de las especies medicinales citadas. De acuerdo con Bermúdez \& Velásquez (2002); estos datos pueden utilizarse como indicadores del grado de consenso en el uso de las especies y de su importancia cultural en las comunidades investigadas. Se utilizaron los siguientes índices cuantitativos:

IVU: se empleó el enfoque de consenso de informantes, metodología desarrollada por Adu-Tutu et al. (1979) y Phillips (1996). Este valor expresa la importancia o valor cultural de una especie determinada para todos los informantes entrevistados. Para estimar el índice de valor de uso general de cada especie para todos los informantes (IVUs), se utilizó la fórmula:

$$
\mathrm{IVUs}=\frac{\sum \mathrm{i} \mathrm{UVis}}{\mathrm{Ns}}
$$

donde: UVis = número de usos mencionados por cada informante (i), para cada especie (s) y Ns = número de informantes entrevistados.

Los datos de cada informante son usados para calcular el número promedio de usos de una especie en particular (Cotton 1999). De esta forma, los usos identificados por cada informante fueron promediados para obtener el índice de valor de uso general para cada especie. Esta estadística se puede aplicar a cualquier técnica de obtención de datos, en la que numerosas personas, proporcionan información sobre la gama de usos de alguna planta (Martin 1995).

UST: esta metodología, expresa que aquellos usos medicinales que son citados con una frecuencia superior o igual al $20 \%$, por las personas encuestadas que usan plantas como primer recurso para un determinado problema de salud, pueden considerarse significativos desde el punto de vista de su aceptación cultural y, por lo tanto, merecen su evaluación y validación científica.

$$
\text { UST = Uso Especie(s) } \mathrm{x} 100 / \mathrm{nis}
$$

donde: Uso Especie $(\mathrm{s})$ = número de citaciones para cada especie. nis = número de informantes encuestados.

\section{RESULTADOS}

Durante la revisión documental sobre los principales trabajos científicos que abordan el tema de las plantas diuréticas, se obtuvo una lista de 179 plantas que la población utiliza con fines diuréticos en Cuba; dichas plantas pertenecieron a 78 familias, siendo su selección más azarosa que a un criterio quimio-taxonómico. Las familias más representadas fueron las Gramíneas o Poaceae con 12 plantas, las Papilionáceas con 11, las Compuestas con 10, las Solanáceas con 6, y las Amarantáceas y Malváceas con 5 plantas, respectivamente. En las otras familias, el número de plantas fue reducido; por ello, se refleja que no hay uniformidad en la composición química de las mismas. Los tipos y cantidad de los metabolitos secundarios responsables de la actividad farmacológica de estas plantas medicinales varían según la familia a la cual pertenecen.

A partir del cuestionario y las entrevistas realizadas se identificó la utilización de 26 especies de plantas diuréticas correspondientes a 19 familias botánicas (Cuadro 1). Las familias más representativas fueron: Cesalpinaceae (3 plantas) y Costaceae, Poaceae, Laminaceae, Lauraceae y Rutaceae (2 plantas), el resto de las especies correspondieron a familias diferentes. El hecho de que solo exista coincidencia para una sola familia de las más representadas en la revisión (Poaceae), se sustenta en que el criterio de selección de las mismas obedece al enfoque etnomédico.

Podemos apreciar también, la gran cantidad de acciones medicinales atribuidas a las plantas estudiadas, pero todas coinciden en su efecto diurético. Algunas de éstas se les conocieron más por otras acciones farmacológicas, pero a pesar de ello se incluyeron en la investigación. Un gran número de plantas con valores elevados de IVUs y UST carecen de validación científica en laboratorios de nuestro país, lo que plantea la necesidad de realizar la comprobación de su efecto a nivel experimental. Se encontraron un total de 14 plantas (53.8\%) carentes de reportes de validación preclínica de su efecto diurético en Cuba, el resto de las 
CUADRO 1

Plantas usadas popularmente como diuréticas en las comunidades de Quemado de Güines

TABLE 1

Medicinal plant Medicinal plants used as diuretics in the communities of Quemado de Güines, Cuba

\begin{tabular}{|c|c|c|c|c|c|c|}
\hline Familia & Especie & $\begin{array}{c}\mathrm{N}^{\circ}{ }^{\circ} \\
\text { Herbario }\end{array}$ & $\begin{array}{c}\text { Partes } \\
\text { utilizadas }\end{array}$ & $\begin{array}{l}\text { N. } \\
\text { citas }\end{array}$ & $\begin{array}{l}\text { Nivel } \\
\text { de uso }\end{array}$ & $\begin{array}{c}\text { Índice de } \\
\text { Valor }\end{array}$ \\
\hline Acantaceae & Justicia pectoralis Jacq. & ULV 9879 & Parte aérea & 14 & 16.4 & 0.164 \\
\hline Alliaceae & Alliun cepa $\mathrm{L}$. & ULV 9377 & Bulbo & 23 & 27.0 & 0.270 \\
\hline Asteraceae & Bidens pilosa $\mathrm{L}$. & ULV 791 & Parte aérea & 51 & 60.7 & 0.607 \\
\hline Anacardíaceae & Manguifera indica $\mathrm{L}$. & ULV 9376 & Hojas & 17 & 20.0 & 0.20 \\
\hline Arecaceae & Roystonea regia O.F. Cook. & ULV 9874 & Raíz & 58 & 68.2 & 0.682 \\
\hline Asteraceae & Parthenium hysterophorus L. & ULV 329 & Parte aérea & 13 & 15.2 & 0.152 \\
\hline \multirow[t]{3}{*}{ Cesalpinaceae } & Cassia alata $\mathrm{L}$ & ULV 9877 & Hojas & 51 & 60.7 & 0.607 \\
\hline & Caesalpinia bahamensis Lam. & ULV 9586 & Corteza & 50 & 58.8 & 0.588 \\
\hline & Tamarindus indica $\mathrm{L}$. & ULV 9882 & Frutos & 44 & 51.7 & 0.517 \\
\hline \multirow[t]{2}{*}{ Costaceae } & Costus pictus D. Don. & ROIG 4710 & Hojas & 20 & 23.5 & 0.235 \\
\hline & Costus cylindricus Jacq. & ULV 9876 & Parte aérea & 58 & 68.2 & 0.682 \\
\hline Commelinaceae & Rhoeo discolour (L’Her.) & ULV 9875 & Parte aérea & 41 & 48.4 & 0.484 \\
\hline Compuestas & Xanthium chinense Mill. & HPVC 4914 & Raíces & 63 & 74.1 & 0.741 \\
\hline Crucíferas & Lepidium virginicum $\mathrm{L}$. & ULV 1373 & Parte aérea & 58 & 68.2 & 0.682 \\
\hline \multirow[t]{2}{*}{ Poaceae } & Zea mays $\mathrm{L}$ & ULV 98881 & Estilos & 39 & 45.8 & 0.458 \\
\hline & Cymbopogon citratus (DC) Stapf. & ULV 9671 & Hojas & 18 & 21.1 & 0.211 \\
\hline Labiaceae & Ocimum basilicum $\mathrm{L}$. & ULV 9427 & Hojas & 9 & 10.5 & 0.105 \\
\hline \multirow[t]{2}{*}{ Lamiaceae } & Coleus amboinicus (Lour.) Spreng. & ULV 9880 & Hojas & 18 & 21.1 & 0.211 \\
\hline & Orthosiphon aristatus Blume. & $*$ & Parte aérea & 64 & 75.2 & 0.752 \\
\hline \multirow[t]{2}{*}{ Lauraceae } & Persea americana Miller. & HPVC 5701 & Hojas & 52 & 61.1 & 0.611 \\
\hline & Nectandra coriacea (Sw.) Griseb. & ULV 9201 & Tallo & 59 & 69.4 & 0.694 \\
\hline Mimosaceae & Dichrostachys cinerea (L.) Wight \& Arn & ULV-9493 & Hojas & 19 & 22.30 & 0.223 \\
\hline Nyctaginaceae & Boldoa purpurascens Cav. & ULV 8326 & Hojas & 67 & 78.8 & 0.788 \\
\hline \multirow[t]{2}{*}{ Rutaceae } & Zanthoxylum fagara $\mathrm{L}$. & ULV 9427 & Corteza & 36 & 42.3 & 0.423 \\
\hline & Citrus aurantium L. & ULV 9878 & Fruto, Hojas & 42 & 49.4 & 0.494 \\
\hline Urticareae & Urera baccifera $\mathrm{L}$. & ULV 7878 & Raíces & 54 & 63.5 & 0.635 \\
\hline
\end{tabular}

plantas han sido estudiadas en varias instituciones del país, pero aún no se ha autorizado su empleo por el Buró Regulatorio para la Protección de la Salud.

Los nombres vulgares ayudaron en la identificación de las plantas en las comunidades estudiadas, pues los yerberos no manejaban el nombre científico de la gran mayoría. La forma de preparación más empleada fue la decocción, pero no se refirió la cantidad de droga seca por mililitros de solución. Se reportó en muchos casos la administración de la infusión de la planta y en otros beberla como agua común, recomendándose adicionar hasta 10 hojas de la planta para preparar el cocimiento. El cuestionario aplicado a conocedores de plantas en cada una de las comunidades estudiadas arrojó gran coincidencia entre las plantas identificadas en la entrevista a yerberos. Los conocedores refirieron que han prescrito plantas diuréticas, fundamentalmente a familiares y vecinos, recomendando su uso por más de una semana, con total desconocimiento de sus contraindicaciones. 
La entrevista focalizada dirigida a los médicos practicantes de medicina tradicional con conocimientos de fitoterapia clínica evidenció que ellos, a diferencia de los conocedores, dominaban los nombres científicos de algunas especies y conocían en muy poca medida su composición química. En relación con la forma de prescripción de las plantas diuréticas, algunos de ellos refirieron indicarlas a los pacientes a pesar de conocer la carencia de validación experimental en algunas de ellas. A pesar de esto, los médicos expresaron que se observaba mejoría en todos los pacientes y no se apreció fracasos terapéuticos.

Los resultados de la triangulación de la revisión documental realizada en las plantas diuréticas, su validación y enfoque etnomédico permitió conocer que del total de plantas reportadas existen muy pocas en las que se ha realizado estudios preclínicos de farmacología y toxicología experimental en Cuba.

Por otra parte la triangulación de los resultados obtenidos a partir de las entrevistas a yerberos y el cuestionario aplicado a conocedores permitió observar varias coincidencias entre los tres instrumentos aplicados respecto a que muchas de las plantas referidas por los yerberos y conocedores coinciden con las encontradas en la literatura científica con este reporte de forma tradicional. Hubo coincidencia entre todos los entrevistados de que estas plantas necesitan ser estudiadas con fines científicos y corroborar el efecto diurético in vivo.

Las plantas más referidas en el estudio que no se han evaluado como diuréticas en Cuba son: Costus pictus D. Don (caña Mexicana), Justicia pectoralis Jacq. (Tilo), Citrus aurauntium L. (Naranjo agrio), Coleus amboinicus (Lour.) Spreng (Orégano francés), Ocimum bacilicum L. (Albahaca blanca), Allium cepa L. (Cebolla blanca), Zanthoxylum fagara L. (Aruña gato), Nectandra coriacea (Sw.) Griseb. (Sigua), Urera baccifera L. (Chichicate), Persea americana Miller (Aguacate), Cassia alata L. (Guacamayón), Parthenium hysterophorus L. (Escoba amarga) y Roystonea regia O.F. Cook (Palma real) y Dichrostachys cinerea (L.) Wight \& Arn (Marabú).
En el cuadro 1, se encuentra la totalidad de los valores correspondientes al nivel de uso significativo para cada una de las especies registradas con su número de citaciones y el uso respectivo en las comunidades estudiadas. En general se presentaron 11 especies usadas como diuréticas y para el tratamiento de afecciones renales con un UST superior al 60\%. Entre ellas se encuentran: Nectandra coriacea (Sw.) Griseb. (Sigua), Urera baccifera L., Persea americana Miller, Cassia alata L., Boldoa purpurascens Cav (Nitro blanco), Bidens pilosa $\mathrm{L}$ (Romerillo blanco) y Costus cylindricus Jacq. (Caña de la India), Xanthium chinense Mill. (Guisazo de caballo), Orthosiphon aristatus Blume. (Té de riñón) y Lepidium virginicum L. (Mastuerzo) y Roystonea regia O.F. Cook.

Independientemente de los factores anteriormente descritos se puede precisar también que algunos de los metabolitos presentes en estas plantas que aparecen referidos en la literatura (flavonoides, saponinas y sales potásicas) pueden justificar su acción diurética. El empleo tradicional de estas plantas con fines diuréticos durante muchos años le confiere sin dudas cierto aval de seguridad y efectividad.

\section{DISCUSIÓN}

Es necesario realizar estudios farmacológicos preclínicos que logren la validación científica de las plantas identificadas. Las especies carentes de comprobación experimental de su efecto in vivo, deben ser estudiadas según la ruta crítica de evaluación de plantas medicinales, para posteriormente iniciar los trámites de inclusión en la Farmacopea Cubana de Plantas Medicinales. Una vez aprobadas para su empleo en humanos pasarán a formar parte del Sistema Cubano de Información en Fitomedicina (Fitomed) (González \& Ramírez 2007).

De las 179 plantas encontradas en la revisión documental, sólo 17 (9\%) han sido evaluadas mediante estudios experimentales usando modelos animales en Cuba, por lo que la existencia de un porcentaje mayor de plantas no evaluadas justifica la necesidad de comprobar científicamente el efecto diurético de las 
mismas para garantizar su uso seguro y eficaz, ya que la atribución de tal propiedad, de forma tradicional, pudiera estar influenciada por su forma de administración (infusión o decocción), que implican la ingestión de un volumen grande de líquidos, pudiendo incrementar la orina excretada, sin que exista realmente una acción diurética (Boffill 2008).

Es importante destacar que algunas de las especies identificadas como carentes de validación científica, han sido estudiadas en otros países de Latinoamérica, contando algunas referencias, entre otras fuentes, en: NAPRALERT (acrónimo de Natural Products Alert) (Universidad de Illinois, Chicago, N.R. Farnsworth Ed.), Medicinal and Aromatic Plants Abstract (Council for Cientific and Industrial Research, CSIR, India), Native American Ethnobotany Database. (Universidad de Michigan, D. Moerman Ed.) y la Phytochemical and Ethnobotanical Database (ARS, USDA, J. Duke Ed.) (Abreu \& Cuellar 2008). Sin embargo estas plantas cuyos estudios no se han realizado en Cuba se han considerado para su validación debido a la influencia de factores como: edad de la planta, clima (temperatura, régimen de lluvias, luz, humedad, latitud, altitud), cultivo y factores biológicos, que pueden modificar la respuesta biológica al influir en la composición química de las plantas medicinales (Miranda \& Cuellar 2000).

Los resultados etnobotánicos encontrados en nuestro estudio coinciden con una investigación desarrollada en la provincia de Camagüey donde se reportaron un total de 111 especies, pertenecientes a 96 géneros y 55 familias de plantas. En dicho estudio se constató que junto a los remedios naturales elaborados con plantas medicinales, se han usado diversos excipientes o vehículos como formas de administración popular, siendo el agua el más referido (Beyra et al. 2008).

Existe coincidencia también entre las familias de plantas encontradas en nuestro trabajo con las propiedades diuréticas atribuidas a las principales familias identificadas en otras investigaciones etnobotánicas realizadas en Cuba (González \& Ramírez 2007, Pimentel
2009). Los nombres vernáculos referidos en el municipio de Quemado de Güines coincidieron también con la gran mayoría de los encontrados en otras provincias del país, donde se obtuvieron 116 nombres vernáculos (González \& Ramírez 2007, Pimentel 2009).

Las plantas recomendadas por el personal médico que carecen de validación experimental coincidieron en la mayoría de los casos con algunas de las utilizadas en el Valle de San Juan de Pinar del Río, donde se listaron más de 30 especies obtenidas de los saberes populares y reportadas en la literatura científica (Pimentel 2009).

Se evidencia que son escasos los cocimientos sobre las formas de preparación de estas plantas por la población como la elaboración de decocciones, pues no se detallan de manera exacta, siendo esta la forma de más frecuente de administración. Este método se usa sobre todo cuando se emplean partes duras de la planta, como las raíces, cortezas, semillas y tallos. No debe usarse si la planta contiene aceites volátiles. Se pone la planta con agua fría, se hierve 10 minutos y se filtra. Se recomienda además echar un poco más de agua para compensar lo que se pierde con la ebullición (González \& Ramírez 2007).

Se encontraron algunas especies con estudios fármaco-toxicológicos realizados en Cuba como: Bidens pilosa L, Carica papaya L (Fruta bomba), Rhoeo spathacea (Sw.) Stearn. (Cordován). (Boffill et al. 2006). Se reportó además la actividad diurética in vivo de Boldoa purpurascens Cav. (Pérez et al. 2008, Pérez et al. 2009), Xanthium chinense Mill. (Jiménez et al. 1999), Lepidium virginicum L. (Carvajal et al. 1986), Orthosiphon aristatus Blume (León et al. 1996) y Tamarindus indica L. (Martínez 2007).

Se identificaron 26 plantas medicinales utilizadas como diuréticas por las comunidades estudiadas, donde 14 de ellas carecían de validación a nivel experimental en Cuba: Costus pictus D. Don, Justicia pectoralis Jacq., Citrus aurauntium L., Coleus amboinicus, Ocimum bacilicum L., Allium cepa L., Zanthoxylum fagara L., Nectandra coriacea (Sw.) Griseb., 
Urera baccifera L., Persea americana Miller, Cassia alata L., Parthenium hysterophorus L., Roystonea regia y Dichrostachys cinerea (L.) Wight \& Arn. Se encontraron 10 plantas con un mayor nivel de uso significativo y un mayor IVU donde se situaron algunas de las no validadas, lo que revela que el conocimiento tradicional continúa profundamente arraigado a las comunidades estudiadas.

\section{AGRADECIMIENTOS}

Los autores agradecen al Proyecto Ramal auspiciado por el Ministerio de Salud Pública de Cuba por el soporte financiero para el desarrollo de la investigación. A dos revisores anónimos que nos dieron sugerencias que ayudaron a perfeccionar el manuscrito.

\section{RESUMEN}

La flora de Cuba es muy rica en plantas medicinales, de las cuales se reportan aproximadamente 179 especies que la población utiliza para fines diuréticos, sin embargo, un gran número de ellas carece de validación experimental de su efecto farmacológico. El presente estudio se realizó con el propósito de documentar la importancia relativa de las especies medicinales más empleadas con fines diuréticos en el municipio de Quemado de Güines de la provincia de Villa Clara, Cuba. Se realizó un estudio etnobotánico en dos comunidades pertenecientes a ese municipio, para lo cual se realizó un muestreo a un total de 85 habitantes, y se aplicó aleatoriamente 80 encuestas a conocedores de plantas y 5 entrevistas entre yerberos y médicos practicantes de medicina tradicional, los cuales nos indicaron las plantas más utilizadas con estos fines en las comunidades estudiadas. La información etnofarmacológica fue registrada mediante la metodología TRAMIL y las especies de interés fueron identificadas y depositadas en el Herbario de la Universidad Central "Marta Abreu" de Las Villas. Los datos se analizaron mediante los índices cuantitativos de valor de uso para cada especie (IVU) y el nivel de uso significativo (UST). Este indicador expresa que aquellos usos medicinales que son citados con una frecuencia superior o igual al 20\%, por las personas encuestadas pueden considerarse significativos desde el punto de vista de su aceptación cultural y, por lo tanto, merecen su validación científica en Cuba. Se identificaron 26 especies medicinales agrupadas en 19 familias botánicas y se encontraron 10 plantas con un mayor nivel de uso significativo y un mayor IVU. El 53.8\% de las plantas reportadas como diuréticas no se han validado experimentalmente en Cuba, el resto de las especies identificadas han sido estudiadas a nivel preclínico, pero aún no está autorizado su empleo como fitomedicamento.

Palabras clave: etnobotánica, plantas medicinales, diuréticos, conocimiento tradicional, Quemado de Güines.

\section{REFERENCIAS}

Abreu, O.A. \& A. Cuellar. 2008. Estrategia en la selección de las plantas medicinal es a investigar. Rev Cubana Plant Med. 13: 30-6. (también disponible en línea: http://scielo.sld.cu/scielo.php?script=sci_ arttext\&pid=S1028-47962008000300009\&lng=es\&n $\mathrm{rm}=$ iso\&tlng=es).

Adu-Tutu, M., Y. Afful, K. Asante-Appiah, D. Leberman \& M. Elvislewis. 1979. Chewing Stick Usage in Southern Ghana. Econ. Bot. 33: 320-328.

Bermúdez, A. \& D. Velásquez. 2002. Etnobotánica médica de una comunidad campesina del estado Trujillo, Venezuela: un estudio preliminar usando técnicas cuantitativas. Rev Fac Farm Univ Cent Venez. 44: 2-6.

Beyra A., M.C. León \& E. Iglesias. 2008. Estudios etnobotánicos sobre plantas medicinales en la provincia de Camagüey (Cuba). Anales del Jardín Botánico de Madrid. 61: 185-204.

Boffil, M.A., G. Lorenzo, E. Monteagudo, M Sueiro \& J. Matos. 2006. Diuretic activity of the medical plants used popularly in Cuba. Pharmacologyonline 3: 435-441.

Boffill, M.A. 2008. Plantas medicinales usadas en Cuba con efecto diurético comprobado experimentalmente. Medicentro Electrónica 11. (también disponible en línea:http://www.vcl.sld.cu/sitios/medicentro/paginas $\% 20 \mathrm{de} \% 20 \mathrm{acceso} / \mathrm{Sumario} / \mathrm{ano} \% 20$ 2008/v12n1a08/plantas81.htm).

Carvajal, D., A. Casacó \& R. González. 1986. Actividad diurética e hipotensora de cuatro especies de plantas con reportes en medicina popular. Rev. CENIC 17: 34-36.

Cotton, C. 1999. Ethnobotany. Principles and Applications, p. 424-32. Baffins Lane, Chichester, West Sussex. John Wiley \& Sons, England.

González, M. \& D. Ramírez. 2007. Antecedentes y situación reguladora de la medicina herbaria en Cuba. BLACPMA 6: 118-24.

Gauniyal, A.K., A.K Rawat \& P. Pushpangadan. 2005. Interactive meeting for evidenced-based complementary and alternative medicines: a report. Evid Based Complement Álternat Med. 2: 249-52. 
Flórez, J. 2003. Farmacología humana. Masson, Barcelona, España.

Jiménez, L., M.C. León, R. Herrera \& G. García. 1999. Efecto diurético del Xanthium strumarium L. (guizazo de caballo). Rev. Cubana Plant. Med. 1: 22-5.

Leon, M.C., J. Tillán, A. Hernández \& J.L Cárdenas. 1996. Efecto diurético y toxicidad aguda del Orthosiphon aristatus Blume (te de riñón). Rev. Cubana Plant. Med. 1: 26-30.

Macías, B.P. 2009. Terapia con plantas medicinales. In Plantas medicinales y embarazo. Editorial Oriente, Santiago de Cuba, Cuba.

Marcia, A., P. Jerome \& M.D. Kassirer. 2005. Medicina alternativa. Los riesgos de remedios no probados, no regulados. N. Engl. J. Med. (también disponible en línea: http://www.homowebensis.com/archivos/ editorial).

Martin, G.J. 1995. Ethnobotany. A method manual. Chapman \& Hall, London.

Martínez-Novellas, Y. 2007. Efecto diurético del Tamarindus indica en ratas. Rev. Cubana Farm. 41: 179.

Miranda, M. \& A. Cuellar. 2000. Farmacognosia y química de los productos naturales. Editorial Félix Varela, La Habana, Cuba.

Morón, F.J. 2002. Plantas medicinales y medicamentos herbarios. In F.J. Morón \& M. Levy. Farmacología general. Editorial Ciencias Médicas, La Habana, Cuba.

Pérez, M., M. Cid, R. Méndez, M. Rodríguez \& M. Arboláez. 2007. Proposal of guideline for clinical trial protocols with herbal drugs. J Biomed. 1: 16-20. (también disponible en línea: http://biomed.uninet. edu/2007/n1/perez.html).
Pérez, M., M. Boffill, D.M. González \& E. Monteagudo. 2009. Efecto de la administración oral continuada de Boldoa purpurascens Cav. sobre diferentes variables fisiológicas en ratas. BLACPMA 8: 204-210.

Pérez, M., E. Monteagudo \& M. Boffill. 2008. Evaluación de la toxicidad aguda de un extracto de Boldoa purpuracens Cav. por el método de las clases. Lat. Am. J. Pharm. 27: 250-4.

Pérez, M., M.L Sueiro, M.A Boffill \& F. Morón. 2011. Estudio etnobotánico de las plantas más utilizadas como diuréticas en la Provincia de Villa Clara, Cuba. BLACPMA 10: 46-55

Phillips, O. 1996. Some Quantitative Methods for Analyzing Ethnobotanical Knowledge. The New York Botanical Garden, EEUU.

Pimentel, E. 2009. Estudio etnobotánico de las plantas medicinales en el Valle de San, Pinar del Río, Cuba. (también disponible en línea: http://www. monografias.com/trabajos71/estudio-etnobotanicoplantas-medicinales/estudio-etnobotanico-plantasmedicinales 3. shtml).

Rang, H.P., M.M. Dale \& J.M. Ritter. 2008. Pharmacology. Elsevier, Philadelphia, EEUU.

Roig, J.T. Nitro. 1998. Plantas medicinales y aromáticas o venenosas de Cuba. Editorial Científico-Técnica. La Habana, Cuba.

TRAMIL. 2006. Requerimientos de encuestas. Programa de investigación aplicada a la medicina popular del Caribe. (también disponible en línea: http://www. funredes.org/endacaribe/TramilEncuestas.html).

Verpoorte, R., Y.H. Choi \& H.K. Kim. 2005. Ethnopharmacology and systems biology: a perfect holistic match. J. Ethnopharmacol. 100: 53-56. 
